\title{
Small distortion and volume preserving embeddings for Planar and Euclidean metrics
}

\author{
Satish Rao \\ NEC Research Institute \\ 4 Independence Way \\ Princeton, NJ 08540
}

\begin{abstract}
A finite metric space, $(S, d)$, contains a finite set of points and a distance function on pairs of points. A contraction is an embedding, $h$, of a finite metric space $(S, d)$ into $R^{d}$ where for any $u, v \in S$, the Euclidean $\left(\ell_{2}\right)$ distance between $h(u)$ and $h(v)$ is no more than $d(u, v)$. The distortion of the embedding is the maximum over pairs of the ratio of $d(u, v)$ and the Euclidean distance between $h(u)$ and $h(v)$.

Bourgain showed that any graphical metric could be embedded with distortion $O(\log n)$. Linial, London and Rabinovich and Aumman and Rabani used such embeddings to prove an $O(\log k)$ approximate max-flow min-cut theorem for $k$ commodity flow problems.

A generalization of embeddings that preserve distances between pairs of points are embeddings that preserve volumes of larger sets. In particular, A $(k, c)$-volume respecting embedding of $n$-points in any metric space is a contraction where every subset of $k$ points has within an $c^{k-1}$ factor of its maximal possible $k-1$-dimensional volume.

Feige invented these embeddings in devising a polylogarithmic approximation algorithm for the bandwidth problem using these embeddings. Feige's methods have subsequently been used by Vempala for approximating versions of the VLSI layout problem.

Feige showed that a $\left(k, O\left(\log ^{3 / 2} n \sqrt{k / \log k}\right)\right)$ volume respecting embedding existed. He recently found improved $(k, O(\sqrt{\log n} \sqrt{k \log k+\log n}))$ volume respecting embeddings.

For metrics arising from planar graphs (planar metrics), we give $(k, O(\sqrt{\log n}))$ volume respecting contractions. As a corollary, we give embeddings for
\end{abstract}

Permission to make digital or hard copies of all or part of this work for personal or classroom use is granted without fee provided that copies are not made or distributed for profit or commercial advantage and that copies bear this notice and the full citation on the first page. To copy otherwise, to republish, to post on servers or to redistribute to lists. requires prior specific permission and/or a fec.

SCG'99 Miami Beach Florida

Copyright ACM 1999 1-58113-068-6/99/06...\$5.00 planar metrics with distortion $O(\sqrt{\log n})$. This gives rise to an $O(\sqrt{\log n})$-approximate max-flow min-cut theorem for multicommodity flow problems in planar graphs.

We also give an improved bound for volume respecting embeddings for Euclidean metrics. In particular, we give an $(k, O(\sqrt{\log k \log D}))$ volume respecting embedding where $D$ is the ratio of the largest distance to the smallest distance in the metric.

Our results give improvements for Feige's and Vempala's approximation algorithms for planar and Euclidean metrics. For volume respecting embeddings, our embeddings do not degrade very fast when preserving the volumes of large subsets. This may be useful in the future for approximation algorithms or if volume respecting embeddings prove to be of independent interest.

\section{Introduction}

A finite metric space, $(S, d)$, contains a finite set of points and a distance function on pairs of points. A contraction is an embedding, $h$, of a finite metric space $(S, d)$ into $R^{d}$ where for any $u, v \in S$, the Euclidcan $\left(\ell_{2}\right)$ distance between $h(u)$ and $h(v)$ is no more than $d(u, v)$. The distortion of the embedding is the maximum over pairs of the ratio of $d(u, v)$ and the Euclidean distance between $h(u)$ and $h(v)$.

Bourgain showed that any graphical metric could be embedded with distortion $O(\log n)$. Linial, London and Rabinovich and Aumman and Rabani used such embeddings to prove an $O(\log k)$ approximate max-flow min-cut theorem for $k$ commodity flow problems.

A generalization of embeddings that preserve distances between pairs of points are embeddings that preserve volumes of larger sets. In particular, A $(k, c)$-volume respecting embedding of $n$-points in any metric space is a contraction where every subset of $k$ poiuts has within an $c^{k-1}$ factor of its maximal 
possible $k$ - 1-dimensional volumc.

Feige invented these embeddings in devising a polylogarithmic approximation algorithm for the bandwidth problem using these embeddings. Feige's methods have subsequently been used by Vempala for approximating versions of the VLSI layout problem.

Feige showed that a $\left(k, O\left(\log ^{3 / 2} n \sqrt{k / \log k}\right)\right)$ volume respecting embedding existed. He recently found improved $(k, O(\sqrt{\log n} \sqrt{k \log k+\log n}))$ volume respecting embeddings.

\subsection{Results}

For metrics arising from planar graphs (planar metrics), we give $(k, O(\sqrt{\log n}))$ volume respecting contractions. As a corollary, we give embeddings for planar metrics with distortion $O(\sqrt{\log n})$. As a corollary, we give embeddings for planar graphical metrics with distortion $O(\sqrt{\log n})$. Combining this result with arguments of Linial, London, and Rabinovich and Aumann and Rabani $[9,1]$, we obtain an $O(\sqrt{\log n})$-approximate $\max$ flow min cut theorem for multicommodity flow problems in planar graphs.

The previous bound of $O(\log n)$ was first derived for grids in 1983 by Karp, et.al. [7], and for general planar graphs in 1993 [8]. In 1994, general graphs were shown to have $O(\log k)$-max flow min-cut theorems for $k$-commodity flow problems $[9,1]$. There are numerous results for finding exact max flow mincut theorems for special cases of the multicommodity flow problem in planar graphs. See, for example, the survey by Frank [6].

We also give an improved bound for volume respecting embeddings for Euclidean metrics. In particular, we give an $(k, O(\sqrt{\log k \log D}))$ volume respecting embedding where $D$ is the ratio of the largest distance to the smallest distance in the metric.

Our Euclidean metric result leads to $\left(k, \log ^{3 / 2} n \sqrt{\log k}\right)$ volume respecting embeddings for general graphs. This follows from the previously mentioned theorem of Bourgain which states that general graphs can be embedded in Euclidean metrics with $O(\log n)$ distortion. This result dominates Feige's when $k=\Omega\left(\log ^{2} n\right)$. We do not, however, know of an application for volume respecting embeddings for this value of $k$.

Our results give improvements for Feige's and Vempala's approximation algorithms for planar graphs and Euclidean metric graphs. For volume respecting embeddings, our results do not degrade very fast when preserving the volumes of large subsets. This may be useful in the future for approximation algorithms or if volume respecting embeddings prove to be of independent interest.

\subsection{Techniques}

Given an Euclidean metric, we construct a coordinate with respect to a paraneter $\delta$ as follows. We place a set of points $R$ uniformly at random in the Euclidean space $^{1}$ so that each point is expected to be at distance $\delta$ from a point in $R$. For each original node, the distance to the nearest point in $R$ is the value for this coordinate. (We refer to the points in $R$ as 0points since the value of the coordinate is 0 on these points.)

We will lower bound the volume of a $k$-point subset $S$ inductively starting with a two node subset $S$ and adding nodes one at a time. The idea is that the $i$ th node that is added looks "random enough" with respect to the previous $i-1$ nodes in many coordinates. Thus, the new node will fall far away from the subspace containing the previous $i-1$ nodes. This implies that the $i$ nodes together have a large volume. (This is essentially the approach of Feige [4].)

Why does the new node look random with respect to the previous nodes? The new node is at least some distance $\Delta$ away from the previous nodes. Consider two nodes $v_{i}$ and $v_{j}$ that are $\Delta$ apart. For the coordinate set with parameter $\Delta / 8$ the closest 0 -points for $v_{i}$ and $v_{j}$ are typically different. Thus, their values are independent. This is the core idea of the result for Euclidean graphs.

For planar graphs, we rely on a partitioning technique for planar graphs developed by Klein, Plotkin and Rao [8]. This technique takes a parameter $\delta$, finds a breadth first search tree in a planar graph, cuts it along levels that are $\delta$ apart, repeats this process on the resulting connected components and then repeats the process a third time on the resulting connected components. This process ensures that two nodes that are $\alpha \delta$ apart will be in separate components for some small enough constant $\alpha$. Coordinates are computed by randomly stretching components and computing distances from the boundaries of the stretched components. Thus, the coordinate's value for a node is independent of the coordinate's value on a node that is $\alpha \delta$ away. Again, we have the independence that we need.

\subsection{Outline}

We proceed by giving some definitions and lemmas from [5]. We then prove our results for planar graphs, and conclude with our results for Euclidean metrics.

\section{Preliminaries}

We restate several definitions and lemmas from [5].

A finite metric space $(S, d)$ contains a finite set $S$ of points and a distance function $d: S \times S \rightarrow$

\footnotetext{
${ }^{1}$ Actually, we do this in a smaller dimensional space derived from the original.
} 
$R^{+}$where $d(i, i)=0, d(i, j)=d(j, i)$, and $d(i, k) \leq$ $d(i, j)+(d j, k)$ for all $i, j, k \in S$.

An embedding of a finite metric space $(S, d)$ in an $L$-dimensional space is a function $\phi: S \rightarrow R^{L}$. Choosing some arbitrary orthonormal coordinate system, we view $\phi$ as a collection of $L$ functions, $\phi_{i}$ : $V \rightarrow R$, for $1 \leq i \leq L$, each specifying a different coordinate in $R^{\bar{L}}$.

For a set of $k$ points $P$ in $R^{L}$, the volume $(\operatorname{Evol}(P))$ is the $k-1$ dimensional $\ell_{2}$ volume of the $k-1$ dimension simplex that has the points of $S$ as its vertices.

The volume of a finite metric space $(S, d)$, $\operatorname{Vol}(S, d)$ (or just $\operatorname{Vol}(S)$, when $d$ is clear from the context), is $\max [\operatorname{Evol}(\phi(S))]$, where the maximum is taken over all contractions $\phi: S \rightarrow R^{|S|-1}$.

The tree volume, $\operatorname{Tvol}(S)$ of a finite metric space $(S, d)$ is $\pi_{e=(i, j) \in T} \dot{d}(i, j)$ where $T$ is the minimum spanning tree of $S$ with cost function $d$.

We will need the following theorem from [5].

Theorem 1 Let $(S, d)$ be a finite metric space with $k$ points. Then

$$
\operatorname{Vol}(S) \leq \frac{T \operatorname{vol}(S)}{(k-1) !} \leq \operatorname{Vol}(S) 2^{(k-2) / 2} .
$$

A $(k, c)$-volume preserving embedding of $(S, d)$ is a contraction where for all $P \subset S$ with $|P|=k$, $\operatorname{Evol}(P) \geq \operatorname{Vol}(P) / c^{k-1}$.

The main technical lemma required by our construction is the following proposition from [5].

Lemma 2 Let $r$ be a random unit vector in $R^{L}$ chosen with spherical symmetry, and let $R^{k}$ be a subspace of $R^{L}$. Let $\ell$ denote the length of the projection of $r$ on $R^{k}$. Then:

- Small projection: for every $0 \leq \epsilon<1, \operatorname{Pr}_{r}[\ell<$ $\epsilon \sqrt{k / L}] \leq(\beta \epsilon)^{k}$, for some universal $\beta>0$.

- Large projections: for $c>1$ and $k=1, \operatorname{Pr}_{r}[\ell>$ $\sqrt{c / L}] \leq e^{-c / 4}$. When $L$ is large, the exponent tends to $-c / 2$ (rather than $-c / 4$ ).

\section{Embeddings for Planar Graphs}

We give a proof that planar graph metrics can be embedded so that they are $(k, \sqrt{\log n})$-volume respecting in Euclidean space. We assume that the graph is unweighted.

\subsection{Forming a coordinate: planar graphs.}

We will form a coordinate with respect to a parameter $\Delta$ for a planar graph $G=(V, E)$.

We define a process A to form $S \subset V$.

\section{Process A:}

1) Take an arbitrary node and build a breadth first tree.

2) Let $d(v)$ for a node $v$ denote its distance from the root in the breadth first tree.

3) Choose a random number $r \in\{0, \Delta\}$, and let $S$ contain all nodes with $d(v) \bmod \Delta=r$.

We form a coordinate as follows.

We use process A to find a subset $S$. We remove $S$ from $G$, and run process A on each connected component of $G-S$ to find subsets whose union we denote by $S^{\prime}$. We use process $A$ once again on each connected component of $G-S-S^{\prime}$, to find subsets whose union we denote by $S^{\prime \prime}$.

For each connected component in $G-S-S^{\prime}-S^{\prime \prime}$, we randomly choose a "rate" uniformly in the interval $[1,2]$. We set the weight of each edge in the connected component to be the rate. For each node in $G$, we compute the distance in the resulting weighted graph to the nearest node in $S \cup S \cup S^{\prime \prime}$; this distance will be the value of this coordinate for the node.

We will give some properties of these coordinates in the lemmas below.

We define a $\delta$-good node to be a node that is at least $\delta$ away from any node in $S, S^{\prime}$ and $S^{\prime \prime}$ in $G$.

Lemma 3 For any node in $G$, there is a constant probability that it is $\frac{\Delta}{10}$-good.

Proof: Recall that process A on a connected component assigns $d(v)$ to each node $v$. The distance of a node to the nearest level chosen to be in $S$ is $|d(v) \bmod \Delta-r|$. This value will be greater than $\frac{\Delta}{10}$ with probability at least $\rho \geq 4 / 5$, since $r$ is chosen uniformly at random from $\{0, \Delta\}$.

The coordinate for $v$ is simply the minimum $(d(v) \bmod \Delta-r) \bmod \Delta$ over three runs of process A. Since the event that $d(v)$ is greater than $\frac{\Delta}{10}$ of each run is at least $\rho$, the event that $d(v)$ is greater than $\frac{\Delta}{10}$ in all three runs is at least $\rho^{3}$.

That is, the probability that the node is $\frac{\Delta}{10}$-good at least a constant.

Lemma 4 For any $\delta$-good node, the coordinate ranges uniformly over an interval $I$ of length at least $\delta$. Moreover, the choice is independent of anything in a different component.

Proof: Recall that the value of the coordinate for a node $v$ is the distance, which we call $d(v)$, to the nearest node in $S \cup S^{\prime} \cup S^{\prime \prime}$ times the rate for the connected component containing $v$. the rate is chosen uniformly over the range $[1,2]$ thus, the value of $v$ ranges uniformly over $[d(v), 2 d(v)]$. For a $\delta$-good node, this interval has length at least $\delta$. 
Moreover, the coordinate's placement in the interval only depends on the choice of the rate for the connected component containing $v$.

Lemma 5 For any two nodes $u$ and $v$, if $d(u, v)>$ $c \Delta$ for a sufficiently large $c$, then $u$ and $v$ will be in separate connected components in $G-S-S^{\prime}-S^{\prime \prime}$.

\section{Proof Sketch:}

This follows directly from Theorem 4.2 in [8]. A corollary of the theorem is restated below.

Corollary 6 If a graph $G$ excludes $K_{3,3}$, then any two nodes $u$ and $v$ that are in the same component of $G-S-S^{\prime}-S^{\prime \prime}$ have $d(u, v)=O(\Delta)$.

\subsection{The embedding}

To form the embedding, $\phi(\cdot)$, we form $\alpha k \log n$ coordinates (for some large enough constant alpha) as described above for each value of $\Delta \in\{1,2, \ldots, D\}$ where $D$ is the maximum distance between any pair of points. We then scale the coordinates by $1 / \sqrt{L}$ where $L$ is the total number of coordinates, i.e., $\alpha k \log n \log D$. (We assume that $D=n$ for the remainder of this section.)

For a point $v, \phi(v)$ is the vector corresponding to the value for $v$ for each of the coordinates produced above.

Since, the difference between the values of two nodes in any coordinate is bounded by their distance we know that the embedding is a contraction; that is the $\ell_{2}$ distance in the embedding is at most $\sqrt{\sum_{k<L}(d(u, v))^{2}} / \sqrt{L} \leq d(u, v)$. a $k$-set.

We proceed by proving a volume lower bound for

Theorem 7 The embedding is $(k, \sqrt{\log n})$ volume respecting for a planar graph.

\section{Proof:}

We prove that the volume of an arbitrary set $S$ of $k$ points is approximately preserved. Recall that the $T \operatorname{vol}(S)$ is $\prod_{e \in M S T(S)} \ell(e)$, where $M S T(S)$ denotes the minimum spanning tree of $S$ in the planar graph metric. We order the points so that any prefix of the ordering is a connected graph in the tree. It is easy to check that for node $i$, the length of the edge connecting it to nodes $1, \ldots, i-1$ in the tree is equal to the minimum distance from node $i$ to any $v_{j}$ with $j<i$. We denote this distance by $q_{i}$.

We will show that for some $\eta=O(\sqrt{\log D})$, the volume is $\prod_{i>2} \frac{q_{i}}{i \eta}$ which in turn is $\frac{V o l(S)}{\eta^{k-1}}$ (by Theorem 1) for $\eta=O(\sqrt{\log D})$.

We assume that inductively the volume of the prefix for $j<i$ is at least $\prod_{i>j \geq 2} \frac{q_{j}}{j \eta}$.
Following Feige's proof [4], it is sufficient to prove the following lemma regarding the embedding $\phi\left(v_{i}\right)$ of the point $v_{i}$.

Lemma 8 With probability at least $1-n^{-3 k}, \mid \phi\left(v_{i}\right)-$ $x \mid \geq q_{i} / \eta$ from an arbitrary node $x$ in the subspace containing $v_{1}, v_{2}, \ldots, v_{i-1}$.

The lemma implies the theorem by the following argument of Feige.

Feige argues if the new point is at least $\delta$ away $\Theta\left(n^{2 k}\right)$ points in the subspace containing $v_{1}, v_{2}, \ldots, v_{i-1}$, then the new point is at least $2 / 3 \delta$ away from the subspace. Specifically, the points are centers of diameter $\delta / 3$ balls that cover the subspace. Thus, the volume of the $i$-simplex is at least $\frac{2 / 3 \delta}{i}$ times the volume of the $(i-1)$-simplex.

The theorem follows since Lemma 8 implies that with probability at least $1-n^{-k}, \phi\left(v_{i}\right)$ is far from any of the $\Theta\left(n^{2 k}\right)$ points.

\section{Proof of lemma:}

We ignore the factor of $\sqrt{L}$ in the coordinates except for the last step.

We consider a set of coordinates that were defined with parameter $\Delta \in\left[q_{i} / 2 c, q_{i} / c\right]$ where $c$ is the constant from Lemma 5 . A constant fraction of these coordinates are $\frac{\Delta}{10}$-good with high probability by Lemma 3 and Chernoff bounds. We focus on the $\frac{\Delta}{10}$-good coordinates for the remainder of the proof.

We know that $x=\sum_{j<i} w_{j} v_{j}$, for some setting of the $w_{i}$ 's. Furthermore, each $v_{j}, j<i$ is in a different component from $v_{i}$ by Lemma 5 . Thus, by Lemma 4 , the value for $v_{i}$ is chosen uniformly from a range of length at least $\frac{\Delta}{10}$ independent of the value of $x$ on this coordinate.

Thus, with probability $\frac{1}{3}$ the value for $v_{i}$ in a $\frac{\Delta}{10}$ good coordinate differs from the value of the coordinate for $x$ by at least $\frac{\Delta}{30}$. (For example, assume that $x$ is in the middle of the range, and that the value of $v_{i}$ is chosen uniformly the interval.)

Recall that there are $\alpha k \log n$ coordinates. $\mathrm{By}$ choosing $\alpha$ to be sufficiently large and using Chernoff bounds, we can show that $\phi\left(v_{i}\right)$ differs from $x$ in at least a constant fraction of the coordinates differ by at least $\Delta / 30$ with probability $1-n^{3 k}$. Thus, $\left|\phi\left(v_{i}\right)-x\right|$ is at least $\Omega(\Delta / 30 \sqrt{k \log n} / \sqrt{L})=$ $q_{i} / \beta \sqrt{\log D}$ for some constant $\beta$, with probability $1-n^{3 k}$.

End of lemma proof.

\section{Embedding Euclidean metrics}

In this section, we take an embedding in $R^{d}$ and produce an embedding into $R^{L}$ where the volume of every $k$-set in the embedding is lower bounded by some function of the tree volume of the $k$-set. Notice that in the original embedding the $k$-set may have zero 
Euclidean volumc. Thus, the problem is not necessarily trivial.

We define an embedding by generating coordinates using the following procedure. We assume for this exposition that the minimum distance between points is at least 1 , and that the maximum distance is $n$. We assume further that $d=\Theta(\log n)$, since we can project to such a space while approximately preserving all distances by a theorem of Johnsonn and Lindenstrass[3].

Coordinate $(P, d, \Delta) /^{*}$ The points $P$ arc in $R^{d}$. */

1. Generate $m=\alpha \log k$ random lines, and project each point onto each line. This generates a mapping of each point into $R^{m}$. (We specify $\alpha$ later.)

2. Let $f$ be the smallest value for any projected point on any projection. Let $l$ be the largest value for any projected point on any projection.

3. Choose $(l-f+2 \Delta)^{m} / \operatorname{Vol}(\Delta, m)$ points uniformly at random in the cube containing all the points in $[l-\Delta, f+\Delta]^{m}$, where $\operatorname{Vol}(\delta, m)$ is the volume of a sphere of diameter $\delta$ in $R^{m}$. We refer to a chosen point as a 0 -point. (That is, we place 0 -points so that each ball of diameter $\Delta$ is expected to contain one 0 -point.)

4. Output for each point in $P$ the distance from its associated point to the nearest 0-point. (We call the points, 0 -points since the value of the coordinate at that point is 0 .)

We now produce an embedding as follows. We assume that the smallest distance is 1 and the largest is $n$.

- Let $m=c \log k$ as in the procedure for forming a coordinate.

- For $l=0$ to $m \log n$

- Let $D_{l}=(1+1 / m)^{l}$.

- For $i=0$ to $k \log n$

(We will generate the level $l$ coordinates)

- Output Coordinate $\left(P, d, D_{l}\right)$

We scale the resulting coordinates by $1 /(\sqrt{k} \log n)$. We refer to the embedding is $\phi$.

Notice that the number of coordinates is $L=$ $\alpha k \log k \log ^{2} n$. Unlike the previous section, the scale factor is larger than $1 / \sqrt{L}$, by a factor of $O(1 / \sqrt{\log k})$ thus the embedding is not necessarily a contraction. In what follows, we prove that the embedding is approximately a contraction as well as lower bounding the volume of every $k$-set. We start by proving the volume lower bound.
Theorem 9 Given an Euclidean metric on $n$ points $P$ into $R^{d}$, the embedding above is a $(k, O(\sqrt{\log k \log n}))$ - volume respecting embedding of the metric.

\section{Proof:}

We will show that the volume of any set $S$ of size $k$ is at least $\operatorname{Vol}(S) /(c \sqrt{\log n \log k})^{k-1}$. We start by considering an ordering $\left\{v_{1}, \ldots, v_{k}\right\}$ of $S$, where $v_{1}$ is chosen arbitrarily and $v_{i}$ is a node in $S-\left\{v_{1}, \ldots, v_{i-1}\right\}$ that minimizes $\min _{m<i} d\left(v_{i}, v_{m}\right)$. We define $q_{i}$ to be the corresponding value.

During the Coordinate procedure, the points are first embedded into an $m=\alpha \log n$-dimensional space. We only consider coordinates where the $\ell_{2}$ distance between the images of two points in $S$ in $R^{m}$ is approximately the same as the original distance between the points. If we choose $\alpha$ to be sufficiently large we can ensure that all the distances are within a factor of two of the original distances in a constant fraction of the coordinates. This follows from the previously mentioned Theorem of Johnsonn and Lindenstrass [3].

We argue inductively that the $i-1$ dimensional volume of the first $i$ points of $S$ is at least

$$
\prod_{1<j \leq i} \frac{q_{j}}{j \gamma \sqrt{\log k \log n}}
$$

for some constant $\gamma$. The theorem follows by using Theorem 1.

The basis is that $\operatorname{Evol}\left(v_{1}, v_{2}\right)=\Omega\left(d\left(v_{1}, v_{2}\right)\right)$ and the embedding is distance preserving which follows from the arguments below.

We consider the coordinates where the distances for all the pairs in $S$ are preserved to within a constant factor in the $R^{m}$ cmbcdding that is used. This is a constant fraction of the coordinates.

Consider a coordinate to be $i$-good when during its formation

1. the 0 -point for $v_{i}$ is different than the 0 -points of each $v_{j}, j<i$,

2. there is exactly one 0-point within distance $D_{l}$ of $v_{i}$ in the projection onto $R^{m}$ used in step 1 of the coordinate procedure, and

3. for each $j<i$, there is at least one 0 -point within distance $2 D_{l}$ of the projection of $v_{j}$ onto $R^{m}$ used in step 1 of the coordinate procedure.

Lemma 10 Any coordinate with $D_{l} \in\left\{q_{i} / 16, q_{i} / 8\right\}$ is $i$-good with constant probability.

\section{Proof of lemma.}

The probability that exactly one point is chosen inside the ball can be computed as follows. The probability that an arbitrary 0 -point hits the ball is

$$
p=\frac{\operatorname{Vol}\left(D_{l}, m\right)}{\left(l-f+2 D_{l}\right)^{m}}
$$


Thus, the probability that exactly one of $q 0$-points hits the ball is

$$
q p(1-p)^{q-1}
$$

Since $q=\frac{\left(l-f+2 D_{l}\right)^{m}}{\operatorname{Vol}\left(D_{l}, m\right)}=1 / p$, this probability is larger than $1 / 4$ as long as $q \geq 2$. Thus, item 2 of the definition of $i$-good holds with constant probability.

Now we prove that item 3 holds with better than constant probability. The probability that a 0 -point hits a ball of diameter $2 D_{L}$ is at least

$$
p^{\prime}=\frac{V o l\left(2 D_{l}, m\right)}{\left(l-f+2 D_{l}\right)^{m}}=2^{m} p .
$$

The probability that no zero point hits a ball of diameter $2 D_{l}$ is at most $\left(1-p^{\prime}\right)^{l} \leq(1 / e)^{2^{m}}=O\left(1 / 2^{k}\right)$. Thus, the probability that $i-1$ balls of diameter $2 D_{l}$ centered at the points $\left\{v_{1}, \ldots, v_{i-1}\right\}$ contain at least one point is at lcast a constant.

Since, $q_{i} \leq D_{l} / 8$, the $2 D_{l}$ diameter balls around centers $\left\{v_{1}, \ldots, v_{i-1}\right\}$ are disjoint from the diameter $D_{l}$ diameter ball around $v_{i}$. Thus, item 1 of the definition of $i$-good holds whenever Items 2 and 3 hold. End of proof of lemma.

As in the proof for our planar graph embedding, we can derive the volume lower bound by using Feige's argument that it is sufficient to show that with probability $1-n^{-3 k}$, that $\phi\left(v_{i}\right)$ is far from an arbitrary node $x$ in the subspace containing $v_{1}, v_{2}, \ldots, v_{i-1}$.

Claim 1 For an arbitrary $x$ in the subspace containing $v_{1}, v_{2}, \ldots, v_{i-1},\left|\phi\left(v_{i}\right)-x\right|>q_{i} / \sqrt{\gamma \log n \log k}$ with probability $1-n^{-3 k}$.

Proof of Claim: For any point in $R^{m}$, the volume of the shell of inner diameter $(1-1 / m) D$ and outer diameter $D$ is a constant fraction of the volume of the sphere of diameter $D$. Moreover, the volume of the sphere of diameter $(1-2 / m) D$ is a constant fraction of the volume of the sphere of diameter $D$.

Thus, for any $i$-good coordinate formed with value $D_{l}$, the value for $v_{i}$ is least $(1-1 / m) D_{l}$ with constant probability. Also, the value is less than $(1-2 / m) D_{l}$ with constant probability.

Using the fact that $x=\sum_{j<i} w_{j} v_{j}$ for some setting of the $w_{j}$ 's, we argues that $v_{i}$ 's value is "independent enough" from the value of $x$ in this coordinate as follows. Each of the values of an $i$-good coordinate for any $v_{j}$ for $j<i$ is set by a point that is outside of the ball of diameter $D_{l}$ that is centered at $v_{i}$. Thus, they cannot affect the position of the 0 -point that sets $v_{i}$ 's value.

Thus, we assume that $x$ 's value is fixed before $v_{i}$ 's value is set. If the value for $x$ is larger than (1$1.5 / m) D_{l}$ then the value for $v_{i}$ differs from $x$ 's value by at least $D_{l} / 2 m$ when it is less than $(1-2 / m) D_{l}$ which occurs with constant probability. Similarly, if the value of $x$ is at most $(1-1.5 / m) D_{l}$, the value for $v_{i}$ differs from $x$ 's value by at least $D_{l} / 2 m$ when it is no more than $(1-2 / m) D_{l}$ which occurs with constant probability.

Thus, the value of $\phi\left(v_{i}\right)$ differs from the value of $x$ by at least $D_{l} / 2 m$ for any $i$-good coordinate with constant probability.

There are $\Omega(k m \log n) i$-good coordinates with $D_{l} \in\left\{q_{i} / 16, q_{i} / 8\right\}$. Thus, the total $\ell_{2}$ difference of $\phi\left(v_{i}\right)$ from $x$ is $\Omega\left(\left(q_{i} / m\right) \sqrt{k m \log n}\right)$ for these coordinates. Multiplying by the scale factor, we get that the difference is at least $\Omega\left(q_{i} / \sqrt{\log k \log n}\right)$ with high probability.

We finish by showing that the embedding is (approximately) a contraction.

Theorem 11 With high probability every $d_{\phi}(i, j)=$ $O((d(i, j))$.

\section{Proof Sketch:}

Claim 2 If $i$ and $j$ have the same 0-point when forming a coordinate $y: V \rightarrow R$ then $\operatorname{Pr}[y(i)-y(j)>$ $d(i, j) \sqrt{c / \log k}]<e^{-c / 4}$.

Notice that $y(i)-y(j)$ is bounded by the length of the projection of the vector from $i$ to $j$ onto the vector from $j$ to the 0 -point. Since the latter vector is a random vector with spherical symmetry, the claim follows from Lemma 2 .

Claim 3 If $i$ and $j$ have different zero points when forming a coordinate $x: V \rightarrow R$ with $\Delta=D_{l}$ then $\operatorname{Pr}\left[y(i)-y(j)>D_{l} c / m\right]<e^{-c / 4}$.

This claim follows from the fact that a ball of diameter $(1-c / m) D_{l}$ has volume that is at most a $e^{-c / 4}$ fraction of the volume of a ball of diameter $D_{l}$.

Claim 4 If a coordinate is formed with $\Delta>$ $\operatorname{cmd}(i, j)$ for nodes $i$ and $j$, then $i$ and $j$ have the same zero point with probability at least $(1-(1 / c))$.

This claim follows from the fact that the intersection of the balls of diameter $c m d(i, j)$ has a volume that is at least a $(1-1 / c)$ times the volume of a $\operatorname{cmd}(i, j)$ ball.

From the 3 claims above we show that with high probability that $d_{\phi}(i, j)$ is $O(d(i, j))$.

For coordinates where $i$ and $j$ have the same zero point the difference has expected value $O(d(i, j) / \sqrt{\log k})$. Moreover, the distribution of the values is favorable by claim 2 . Specifically, by claim 1 and Chernoff bounds, the number of coordinates with value greater than $>d(i, j) / \sqrt{K \log k}$ is $O\left(\log n+L / 2^{K}\right)$ with high probability.

Thus, the total $\ell_{2}$ sum over the coordinates is bounded by $O(d(i, j) \sqrt{k} \log n)+O(d(i, j) \sqrt{\log n})$. The first term comes from the fact that there are at 
most $k \log ^{2} n \log k$ coordinates and from summing a geometric series. The second term comes from the fact that no coordinate is larger than $d(i, j)$.

Coordinates where $i$ and $j$ have different zero points are divided according to the whether $\Delta<$ $d(i, j), \Delta \in\{d(i, j), m d(i, j)\}$ or $\Delta>d(i, j)$.

For the former the expected value is $O(d(i, j) / \log k)=O(d(i, j) / \sqrt{\log k})$ with a favorable distribution by claim 3 . We can argue here as in the case for nodes with the same 0-point.

The contribution for coordinates with $\Delta \in$ $\{d(i, j), m d(i, j)\}$ is at most $d(i, j) \sqrt{k \log n \log k}$ since the range will be dominated by the coordinates where $\Delta$ within a constant factor $m d(i, j)$. There are only $O(k \log n \log k)$ coordinates with $\Delta$ being within a constant factor $m d(i, j)$, thus the total $\ell_{2}$ norm of these coordinates is $O(d(i, j) \sqrt{k \log n \log k})$ which is $O(d(i, j) \sqrt{k} \log n)$.

We argue that the total contribution from coordinates with $\Delta>m d(i, j)$ and differing zero points is small using claim 4 as follows. The total number of coordinates with differing 0-points is approximately $1 / c$ of the coordinates for $\Delta>c m d(i, j)$. Thus, we can bound the contribution by

$$
\sqrt{\sum_{l>l_{0}} \frac{k \log n\left(d(i, j)^{2}\right)}{(1+1 / m)^{l-l_{0}}}}
$$

where $l_{0}$ is the lowest integer where $(1+$ $1 / m)_{0}^{l} \geq m d(i, j)$. The sum is bounded by $O(d(i, j) \sqrt{k \log n \log k})$ which is $O(d(i, j) \sqrt{k} \log n)$.

Thus, after dividing by the scale factor $\sqrt{k} \log n$, we have shown that $d_{\phi}(i, j)$ is $O(d(i, j))$.

\section{References}

[1] Y. Aumann and Y. Rabani. An $O(\log k)$ approximate min-cut max-flow theorem and approxiwation algorithn. SIAM Journal on Computing, 27(1):291-301, 1998.

[2] j. Bourgain. On Lipschitz embedding of finite metric spaces in Hilbert space. Israel J. Math, 52:46-52, 1985.

[3] P. Fankl and H. Maehara. The JohnsonLindenstrauss lemma and the sphericity of some graphs. Journal of Combinatorial Theory, Series $B, 44: 355-362,1988$.

[4] U. Feige. Improved volume respecting embeddings.

[5] Uriel Feige. Approximating the bandwidth via volume respecting embeddings. In Proceedings of the 30h Annual ACM Symposium on Theory of Computing, 1998.
[6] A. Frank. Packing paths, circuits and cuts - a survey. In B. Korte, L. Lovász, H.J. Prömel, and A. Schrijver, editors, Paths, Flows, and VLSILayout, pages 47-100. Springer-Verlag, Berlin, 1990.

[7] Richard M. Karp, Frank Thomson Leighton, Ronald L. Rivest, C. D. Thompson, Umesh V. Vazirani, and Vijay V. Vazirami. Global wire routing in two-dimensionalarrays. In Proceedings of the 24th Annual Symposium on Foundations of Computer Science, pages 453-459, 1983.

[8] P. Klein, S. Rao, and S. Plotkin. Excluded minors, network decomposition, and multicommodity flow. In Proceedings of the 25th $A n$ nual ACM Symposium on Theory of Computing, pages 682-690, May 1993.

[9] N. Linial, E. London, and Y. Rabinovich. The geometry of graphs and some of its algorithmic applications. Combinatorica, 15:215-245, 1995.

[10] Santosh Vempala. Approximating vlsi layout problems. In Proceedings of the 39th Annual Symposium on Foundations of Computer Science, 1998. 